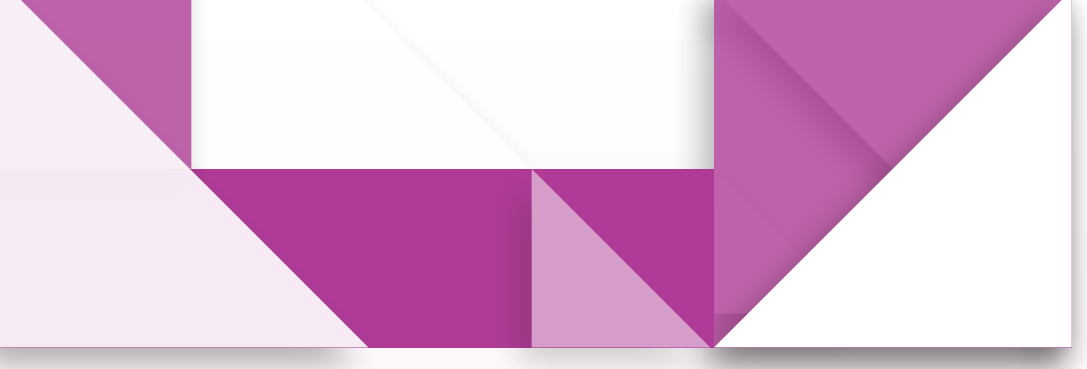

\title{
Catalizando aprendizajes: estrategias metodológicas basadas en las propuestas CTS y ESPC para la enseñanza de la catálisis
}

- Catalyzing Learnings: Methodological Strategies Based on the CTS and ESPC Proposals for Teaching Catalysis

- Catalisando aprendizagens: estratégias metodológicas baseadas nas propostas CTS e ESPC para o ensino da catálise

\section{Resumen}

El presente trabajo corresponde al reporte de un caso educativo sobre educación superior. Para este caso se ha elegido el concepto de catálisis, ya que el $90 \%$ de los productos químicos industriales y usados por la sociedad, son producidos con ayuda de catalizadores. El trabajo se desarrolló en el semillero de investigación del grupo de catálisis de la Universidad del Cauca, a través del estudio de una problemática social referida al calentamiento global, el certificado de emisiones de gases contaminantes en los automóviles, el cual está relacionado directamente con el uso del convertidor catalítico y permitió concluir que el uso de estrategias basadas en CTS y ESPC pueden hacer que la enseñanza de la catálisis tenga sentido para los estudiantes, ya que genera un gusto por aprender, además les permite conocer las implicaciones sociales que tiene el conocimiento.

Palabras clave

catálisis; enseñanza de las ciencias; CTS; ESPC
Jimena Sandoval Sarrias' Alfonso Enrique Ramírez Sanabria ${ }^{2}$

Licenciada en Educación de la Universidad Pedagógica Nacional. Magíster en Educación, línea de la Ciencias Naturales y de las Tecnologías de la Universidad del Cauca, 2014.

ximena9090@gmail.com

2 Licenciado en Biología-Química, de la Universidad del Valle (1993). Magíster en Química de la Universidad del Valle (1998). Docteur en Chimie Appliquée de la Universidad de Poitiers (2007).

aramirez@unicauca.edu.co

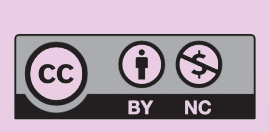




\section{Abstract}

The present work corresponds to the report of an educational case at the Higher Education level. In this work, we have chosen the Catalysis concept, since about $90 \%$ of industrial chemicals that are used by the society, are produced by this way. The work, was developedin Catalysis research Group at University of Cauca, through the study of social problems related to global warming, Vehicule Greenhouse Gas Emissions Standards Program, which is related directly to the use the catalytic converter and allowed to conclude that the use of strategies based on STS and CPS can make the teaching of Catalysis meaningful for students, since it generates a taste for learning, it also allows them to know the social implications of knowledge and in this way learning will have an impact on his acting in the world

Keywords

catalysis; Learning natural sciences; STS; CPS

\section{Resumo}

O presente trabalho corresponde ao relato de um caso educacional no nível do Ensino Superior. Para este caso, Catálise foi escolhida, já que $90 \%$ dos produtos químicos industriais utilizados pela sociedade são produzidos com a ajuda de catalisadores. $\bigcirc$ trabalho foi realizado no laboratório de pesquisa do grupo Catalysis da Universidade do Cauca, através do estudo de um problema social relacionado ao aquecimento global, o certificado de emissões de gases polventes em carros, que está diretamente relacionado ao uso do conversor catalítico e permitiu concluir que o uso de estratégias baseadas no CTS e SPS pode tornar o ensino da Catálise significativo para os alunos, pois gera um gosto pela aprendizagem, também permite que eles conheçam as implicações sociais do conhecimento e, dessa forma, a aprendizagem terá um impacto em sua atuação no mundo

Palavras-chave

catálise; ensino em ciência; CTS; SPS 


\section{Introducción}

Teniendo en cuenta los desafíos que impone el nuevo paradigma de la sociedad del conocimiento y la innovación, es la educación la que debe contribuir a desarrollar la capacidad analítica, innovadora, de reflexión y de comprensión en el futuro ciudadano y profesional (Chaparro, 1998, p. 12). Sin embargo, en la actualidad la enseñanza de las ciencias no está respondiendo del todo a estos desafíos, por lo que se hace necesario plantear opciones para abordar esta enseñanza. En este sentido, los estudios en ciencia, tecnología y sociedad CTS (Corchuelo, Catabiel y Cucuñame, 2006) en el marco de los estudios de situaciones problemáticas contextualizadas (ESPC) (Carrascosa, Gil y Valdés, 2005) brindan una alternativa en la enseñanza de conceptos en ciencias.

Otro aspecto importante a tener en cuenta en este contexto de incertidumbre es la escogencia de los contenidos a trabajar, el cual dependerá en gran medida del impacto que tengan en la sociedad y, para este caso, la catálisis es una de las disciplinas más importantes en la actualidad ya que, entre otros, 1) permite desarrollar procesos para la producción de combustibles y compuestos químicos más eficientes y más limpios, 2) las transformaciones indispensables en la química de los seres vivos requiere catalizadores naturales como las enzimas y 3) esta contribuye a la descontaminación del ambiente (Ramírez, Vargas, Valderruten y Zuluaga, 2008, p. 10).

Teniendo en cuenta lo anterior, en el marco del trabajo de investigación titulado La catálisis en la sociedad: una mirada desde la educación, que se está llevando a cabo actualmente y que pretende encontrar elementos que permitan hacer de la enseñanza de la catálisis un acto que tenga sentido para los estudiantes en cualquier nivel educativo. Para ello, se llevaron a cabo una serie de actividades basadas en las propuestas CTS y ESPC de contextualización, presentación de la situación, búsqueda de conceptos, construcción de relaciones y conclusiones, que permitieron a los jóvenes de un semillero de investigación relacionar el concepto de catálisis con elementos de su contexto, en este caso, la contaminación del aire producto de la combustión interna en los automóviles.

\section{Referentes teóricos}

\section{¿Qué es la catálisis?}

La catálisis se podría entender como un proceso en el cual se aumenta la velocidad de una reacción química. Los catalizadores son materiales que aceleran las reacciones químicas $\sin$ que el catalizador se consuma, son materiales que inducen el cambio, más específicamente, los catalizadores son materiales que cambian la tasa de consecución del equilibrio químico sin que ellos mismos se cambien o se consuman en el proceso. Los catalizadores también proporcionan selectividad o especificidad a los productos particulares que son más deseados (Armor, 2008).

Todos estos atributos sobre la catálisis y los catalizadores se traducen en ahorro de energía, menos contaminación, menos productos secundarios, menores costos en los reactivos y, finalmente, la reducción de emisión de gases con efecto invernadero sobre la atmósfera. Debido a estas características, actualmente de este proceso químico dependen cuatro sectores de la economía mundial: el petróleo, la producción de energía, la obtención de productos químicos y la industria alimentaria, que en conjunto representan más de 10 billones de dólares del PNB del mundial (ACS, 1996). 


\section{¿Qué es educación con sentido?}

Se entiende por la educación con sentido desde los planteamientos de la Unesco en el marco de los llamados "pilares del aprendizaje del siglo XXI" (2006, p. 132), donde se enuncia de la siguiente manera: una educación con sentido requiere que el estudiante obtenga la capacidad de razonar y de ser consciente del impacto que genera el aprendizaje para su actuar en el mundo, y para eso se plantea la necesidad de aprender a aprender, lo que tendrá sentido solo en función de generar un conocimiento que pueda aprovecharse socialmente. Para esto se requiere formar ciudadanos que comprendan las implicaciones sociales del conocimiento, que tengan la capacidad para aprovechar las oportunidades que el nuevo entorno genera, y puedan adaptarse y responder con éxito a los cambios en dicho entorno y a los desafíos y peligros que él encierra (Chaparro, 1998, p. 13).

Por esto se hace necesario preguntarse por el sentido de la educación en ciencias, lo que requiere plantearse la siguiente pregunta: ¿̇cómo enseñar a aprender de otra manera a los estudiantes? Esto implica darle sentido al aprendizaje: ¿̇por qué y para qué se aprende algo? Implica, además, la autorregulación y el fomento del placer por aprender, la pasión por conocer. Al encontrar sentido, el aprendizaje deja de ser sacrificio y el esfuerzo puede incorporar el placer de conocer (Olivé, 2006, p. 50).

\section{¿Por qué el enfoque ciencia, tecnología y sociedad (CTS)?}

El enfoque CTS nació de la necesidad de analizar los aspectos sociales de la ciencia y la tecnología, tanto en lo que refiere a los factores sociales que influyen en el cambio científico y tecnológico, como en lo relacionado con las consecuencias sociales, políticas, económicas, éticas y ambientales de su uso.

De esta manera, sus principales objetivos (Fourez, 1994 citado por Corchuelo et al., 2006, p. 69) están de acuerdo con el interés de la presente propuesta, estos son: (1) preparar a los estudiantes para usar las ciencias y la tecnología en el entendimiento y mejoramiento de su vida diaria; (2) aplicar el conocimiento científico a la vida cotidiana; (3) introducir las implicaciones sociales y ambientales del desarrollo científico y tecnológico; (4) hacer énfasis en todos los niveles educativos sobre la relevancia social y humana de las ciencias y la tecnología. En este enfoque se encuentra una oportunidad de contribuir a la construcción de una enseñanza en ciencias con sentido, objetivo que guía la presente propuesta.

\section{¿Por qué el estudio de situaciones problemáticas contextualizadas (ESPC)?}

El ESPC se caracteriza por ser un proceso de enseñanza y aprendizaje fundamentado en elementos de la investigación guiada u orientada, promovida por el grupo integrado por Jaime Carrascosa, Daniel Gil y Pablo Valdés (Carrascosa et al., 2005); Mariano Martín Gordillo y Juan Carlos González son miembros del grupo Argo y profesores de la cátedra CTS de la Universidad de Oviedo 
(Corchuelo, 2007, p. 212). En el contexto nacional y regional ha sido puesto en práctica por el grupo de investigación del profesor Miguel Hugo Corchuelo en la formación de ingenieros físicos (Corchuelo, 2007) y en el contexto de educación media (Corchuelo et al., 2006).

El ESPC implica el desarrollo, la planeación y ejecución de actividades, entre otras de sensibilización, contextualización, exploración de conceptos, análisis y construcción de relaciones, conclusiones y reflexiones. En cuanto a la selección y organización de los contenidos, se tienen en cuenta las características e intereses de alumnos y docentes, el contexto y la pertinencia de los propios contenidos. Se propone organizar experiencias de aprendizaje con base en métodos y contenidos con los que las ciencias y la tecnología buscan resolver los problemas vitales, individuales y sociales, teniendo en cuenta que el aprendizaje y la enseñanza se justifican y planifican discursiva y progresivamente entre profesores y estudiantes (Klafki, 1986, citado por Corchuelo, 2007, p. 219).

\section{Metodología}

En la práctica, esta propuesta se desarrolló a partir del análisis de datos obtenidos de la recopilación histórica de la catálisis y de los diarios de campo de la observación participante realizada en el semillero de investigación del Grupo de Catálisis del Departamento de Química de la Universidad del Cauca, del que hacen parte 29 jóvenes, de los cuales 21 son mujeres y 8 hombres, de edades entre los 16 y 31 años; de estos, 25 estudian química, 2 ingeniería ambiental, 1 ingeniería civil y 1 antropología, además, 2 jóvenes son investigadores, 2 son estudiantes de la Maestría en Ciencias Químicas y 1 es estudiante de la Maestría en Educación, con la coordinación de 3 docentes del departamento.
Así se tomaron como base las propuestas CTS y ESPC adaptándolas a las necesidades y contextos puntuales del grupo. Se requirió de la planeación de una serie de elementos previos como el lugar, el tiempo, el número de participantes, el material de apoyo, etc.

En la primera actividad se escogió una técnica de participación llamada "juego de roles" como estrategia metodológica para el análisis de relaciones entre la catálisis, y una problemática del contexto actual relacionada con el certificado de emisiones de gases contaminantes en los automóviles; en este seminario-taller se realizó la actividad de sensibilización a través de una representación en la que un ciudadano va por una avenida y es detenido por un agente de tránsito que se encuentra haciendo una revisión del certificado de gases contaminantes y encuentra que el auto de este ciudadano está emitiendo gases por encima de los niveles permitidos y que, además, tiene un certificado de gases de una empresa no autorizada. Esto genera una discusión por la pregunta que el policía hace: "señorita, Żhace cuánto tiempo tiene ese convertidor catalítico?, para lo cual el ciudadano no tiene ninguna respuesta pues, para empezar, no sabe qué es un convertidor catalítico y realmente no le interesa, solo quiere que no le inmovilicen su auto ni le cobren la multa por la infracción, así que decide sobornar al policía y luego de que el policía acepta el soborno se va a su casa preguntándose si realmente valdrá la pena cambiar ese aparato llamado "convertidor catalítico".

De esta manera, se da inicio a la actividad de contextualización en la que se organizó el grupo por equipos y a cada uno se le asignó la representación de uno de los actores cuya intervención fue importante en una discusión pública: comunidad, industria, ambientalistas, Gobierno y academia. Cada equipo a partir 
de una información recopilada, como datos sobre el convertidor catalítico, su funcionamiento, su precio, reacciones químicas que ocurren dentro de él, legislación colombiana sobre las emisiones de gases, metales que conforman el convertidor: su extracción, precio, su recuperación, entre otros. Sobre esta base se organizó un documento en el que se condensó esta información para que los grupos de discusión armaran sus argumentos.

A partir de allí, y después de un tiempo, cada grupo construyó argumentos relevantes para asumir el papel que le correspondió para presentar sus argumentos, primero, exponiendo su visión y competencia dentro de la problemática particular, y luego escuchando y contraargumentando, si era el caso, los planteamientos de los otros equipos. Esta fue la actividad de análisis y construcción de relaciones realizada con el fin de obtener unas conclusiones y reflexiones, que se discutirán más a fondo en los resultados.

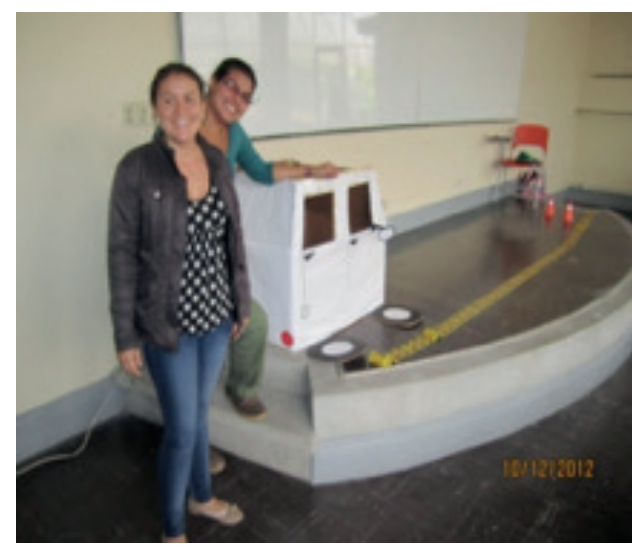

(a)

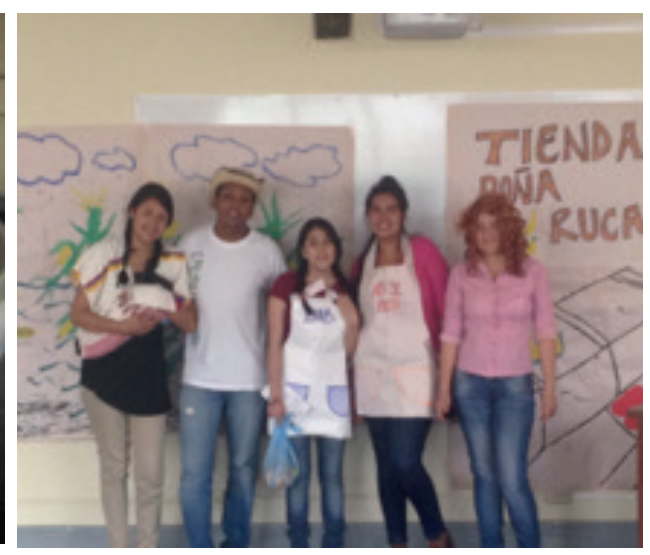

b)

Figura 1. Actividades en el marco de CTS y ESPC. a) Certificado de emisiones de gases contaminantes en los automóviles y b) El calentamiento global y la catálisis

Fuente: elaboración propia.

De acuerdo con la sistematización de los resultados de la actividad anterior, en otra sección de organizó una segunda actividad titulada El calentamiento global y la catálisis; en este caso y a modo de debate frente a la contaminación del aire, la contextualización de la situación fue realizada por algunos miembros del grupo, quienes realizaron un sociodrama en el que mostraron una situación en la que una familia campesina se veía afectada por las lluvias que no correspondían a la época por la que se han guiado tradicionalmente, ayudados por el Almanaque Bristol para sembrar sus productos. Para ellos esta situación desencadena una escasez y alza en los precios de algunos productos de la canasta familiar, en la que los ciudadanos de la zona urbana también se ven afectados.

A partir de esta actividad se planteó la contextualización en el marco de una presentación de imágenes del efecto en nuestro país del calentamiento global, en los últimos años, y luego se aclaran algunos puntos frente a conceptos como 
efecto invernadero, calentamiento global, cambio climático, Protocolo de Kioto, entre otros. Finalmente, se lanza una pregunta detonadora: ¿̇cómo podría contribuir la catálisis al Protocolo de Kioto para el contrarrestar el calentamiento global?

De allí se genera un panel de participación en el que se hace un análisis de la situación y se construyen relaciones entre el conocimiento sobre catálisis y la problemática planteada. De allí surgen elementos y reflexiones que se discutirán a continuación.

\section{Resultados}

Frente a la primera actividad, en las discusiones que se dieron entre los diferentes actores surgieron algunos elementos para tener en cuenta: en primera instancia, la manera de presentar la problemática y la dinámica; por otro lado, el juego de roles generó un interés y una motivación, cada grupo se apropió del rol que cumple en la sociedad y al respecto presentaron sus puntos de vista, la comunidad manifestó su desconocimiento frente a qué es un convertidor catalítico, por lo que deseaban escuchar a los demás antes de plantear su punto de vista (GDT2p17) ' esta posición podría homologarse al común de la ciudadanía que a pesar de usar un automóvil desconoce qué es este elemento y cuál es su función.

Por otro lado, hubo participantes que no lograron establecer relaciones importantes entre los saberes, los conocimientos científicos que se espera que dominen y la problemática planteada, es el caso de los que representan a los ambientalistas, quienes plantean la posición de la necesidad de cambiar el convertidor, pero sus argumentos son muy pobres teóricamente, por ejemplo, manifestaron:

1 La codificación indica (gdł3p57) gd grupo de discusión, t taller, número del taller y p párrafo. sin convertidor habría muchas emisiones de gases al ambiente, los cuales causan afecciones a la salud como problemas respiratorios, por el contrario con el convertidor se tendrá una vida más saludable y una ciudad más limpia. (GDT2p19).

Sin embargo, uno de los participantes de este grupo hizo una reflexión frente a la necesidad de tomar conciencia y de asumir la responsabilidad de tener carro y los efectos que este puede tener en el ambiente, como el calentamiento global, además, citó algunos artículos de la legislación colombiana frente a los límites permitidos (GDT2p20) esta posición va un poco más allá y se acerca al objetivo de los entes ambientales en la sociedad y es ser consciente del impacto que generan las acciones humanas en el medio ambiente, para que en consecuencia se tomen mejores decisiones, además se crea una relación de esta situación con los cambios climáticos que suceden en la actualidad.

Como un tercer elemento se presentan algunos planteamientos con propuestas de solución desde la catálisis, es el caso de los representantes del Gobierno y de la industria, quienes plantean la necesidad de construir convertidores catalíticos más baratos, es decir, utilizando catalizadores que no sean tan costosos como los que se utilizan actualmente y que además sean más eficientes, por otro lado, se argumenta que hoy en día hay que importarlos y sería posible hacerlo en Colombia. De esta manera, el Gobierno y la industria plantean un apoyo a la investigación con el fin de lograr estos objetivos (GDT2p22). Al contrastar estos aportes con el actual sistema general de regalías se observa el apoyo económico que se le está inyectando a la investigación en Colombia, por lo que se puede pensar en hacer de esta propuesta una realidad, pues es un campo de acción de buenas oportunidades en la búsqueda de catalizadores que reemplacen los usados actualmente en los convertidores catalíticos de los automóviles. 
Allí también se apoyan los argumentos del grupo de la academia, que son los únicos que responden a las inquietudes de la comunidad y les explican que el convertidor catalítico es un componente del motor de combustión interna que sirve para el control y reducción de los gases nocivos ( $\mathrm{CO}, \mathrm{C}_{x} \mathrm{H}_{y^{\prime}} \mathrm{NO}$ y los $\mathrm{NO}_{2}$ ) expulsados por el motor de combustión interna. Ellos plantean que la alternativa es usar autos que no usen combustibles fósiles, como, por ejemplo, los carros eléctricos y ojala los sistemas masivos también tengan este tipo de sistemas. Además, solicitan recursos del Gobierno para apoyar la investigación y mejorar estos procesos o proponer alternativas más económicas y ecológicas (GDT2p29).

Al terminar este ejercicio se hicieron algunas reflexiones y aparecieron conclusiones frente a la actividad, en la que los participantes manifestaron que se sentían cómodos y que fue divertido tomar el rol de un ente social, además que es interesante la manera como se presentó la situación ya que no es la dinámica normal de los seminarios abordar problemáticas de la vida cotidiana (GDT2p32). Por otro lado, uno de los docentes hizo un aporte importante frente al gran impacto que tienen los convertidores en el mundo de hoy y cómo ha aportado la catálisis y continúa aportando a mejorar la calidad de vida de la sociedad.

Teniendo en cuenta los argumentos discutidos en esta actividad, se encuentran algunos elementos que se considera importante profundizar, como por ejemplo, qué otros aportes tiene la catálisis frente al calentamiento global. También, se programó la segunda actividad en la que hay elementos para discutir: en primera instancia, la manera de presentar la situación generó en los participantes un espacio de relajación y de diversión que permitió iniciar de un modo diferente el espacio del seminario. Sin embargo, los aportes puntuales de la catálisis para contrarrestar el calentamiento global vinieron principalmente de los docentes, quienes hablaron de elementos como captura de dióxido de carbono con carbón activado, generación de gas de síntesis a partir de dióxido de carbono, energías alternativas utilizando celdas de combustible mejoradas con catalizadores, obtención de energía a partir de hidrógeno, descontaminación de aguas utilizando la electrocátalisis, disminución de solventes que contaminan sintetizando sustancias químicas con catalizadores más eficientes (GDT3p45; GDT3p75; GDT3p77) entre otras, en contraste con la mayoría de los estudiantes que dieron aportes generales como la necesidad de generar nuevos catalizadores para reducir el gasto energético o para generar combustibles alternativos. No obstante, un joven investigador habló del convertidor catalítico y su aporte en la disminución de algunos de los gases de invernadero y un estudiante de maestría habló sobre el uso de zeolitas para capturar $\mathrm{CO}_{2}$ (GDT3p57; GDT3p65).

De manera general, se observó la construcción de pocas relaciones entre la catálisis y la situación planteada en comparación con la primera actividad, esto puede deberse, en gran medida, a la estrategia utilizada pues el ejercicio de los juegos de rol les permitió a los participantes generar mejores aportes. Esta situación produjo una reflexión planteada por uno de los docentes, quien 
manifestó que estaba preocupado porque las respuestas parecen más políticas que académicas, y que al parecer la catálisis se está convirtiendo para ellos en el concepto de paz: todos opinan de este pero nadie da soluciones (GDT3p44; GDT3p52; GDT3p85).

\section{Conclusiones}

Las ESPC con enfoque CTS permiten abrir espacios de discusión en contextos de enseñanza, ya que son actividades desafiantes para los participantes, debido a que se requiere usar el conocimiento adquirido en la comprensión de problemáticas y en el planteamiento de soluciones de contexto.

Se observa que el uso de estrategias basadas en ESPC y CTS pueden hacer que la enseñanza de la catálisis tenga sentido para los estudiantes, ya que genera gusto por aprender, además, les permite conocer las implicaciones sociales que tiene el conocimiento; de esta manera, el aprendizaje tendrá un impacto en su actuar en el mundo.

El trabajo en los semilleros de investigación genera otro tipo de miradas educativas que pueden contribuir al mejoramiento de la enseñanza, en este caso de la catálisis, en ámbitos como el de educación media.

\section{Referencias}

American Chemical Society (ACS). (1996). Report, technology vision 2020, the chemical industry, December.

Armor, J. (2008). What is catalysis or catalysis, so what? The North American Catalysis Society (NACS). globalCatalysis.com

Armor, J. (201 1). A history of industrial catalysis. Catalysis Today (163), 3-9.
Carrascosa, J., Gil, D. y Valdés, P. (2005). ¿Cómo promover el interés por la cultura científica? Santiago: Orealc/Unesco.

Corchuelo, M. (2007). Un giro en la educación en ingeniería (Tesis doctoral). Rudecolombia Universidad del Cauca, Popayán.

Corchuelo, M., Catabiel, V. y Cucuñame, N. (2006). Las relaciones ciencia, tecnología, sociedad y ambiente en la educación media. Popayán: Editorial Universidad del Cauca.

Chaparro, F. (1998). Haciendo de Colombia una sociedad del conocimiento, conocimiento, innovación y construcción de sociedad, una agenda para la Colombia del siglo XXI. Colciencias. Bogotá.

Olivé, L. (2006). Los sentidos de la educación, Orealc/Unesco, Prelac. N ${ }^{\circ} 2$, Chile. Pp. g. 50. 132, 142.

Ramírez, A., Vargas, L., Valderruten, N. y Zuluaga, M. (2008). Boletín vri Vicerrectora de Investigaciones, ed. 16, 9-11.

\section{Para citar este artículo}

Sandoval, J. y Ramírez, A. E. (2019). Catalizando aprendizajes: estrategias metodológicas basadas en las propuestas CTS y ESPC para la enseñanza de la catálisis. Tecné, Episteme y Didaxis: TED, 45, 213-221. 